\title{
Influenza Activity — United States, September 30-December 1, 2018
}

Alicia P. Budd, $\mathrm{MPH}^{1}$; Anwar Isa Abd Elal ${ }^{1}$; Noreen Alabi, MPH${ }^{1}$; John Barnes, PhD ${ }^{1}$; Lenee Blanton, $\mathrm{MPH}^{1}$; Lynnette Brammer, MPH ${ }^{1}$; Erin Burns, $\mathrm{MA}^{1}$; Charisse N. Cummings, $\mathrm{MPH}^{1}$; Vivien G. Dugan, $\mathrm{PhD}^{1}$; Shikha Garg, MD ${ }^{1}$; Rebecca Garten, PhD ${ }^{1}$; Lisa A. Grohskopf, MD ${ }^{1}$; Larisa Gubareva, $\mathrm{PhD}^{1}$; Krista Kniss, $\mathrm{MPH}^{1}$; Natalie Kramer ${ }^{1}$; Alissa O’Halloran, $\mathrm{MSPH}^{1}$; Wendy Sessions, $\mathrm{MPH}^{1}$; Calli Taylor, MPH ${ }^{1}$; David E. Wentworth, PhD ${ }^{1}$; Xiyan Xu, MD ${ }^{1}$; Alicia M. Fry, $\mathrm{MD}^{1}$; Jacqueline Katz, $\mathrm{PhD}^{1}$; Daniel Jernigan, $\mathrm{MD}^{1}$

Influenza activity in the United States was low during October 2018, and, although it increased slowly during November, activity remains low across most of the country.* During the week ending December 1, 2018, the percentage of outpatient visits for influenza-like illness ${ }^{\dagger}$ (ILI) was equal to the national baseline ${ }^{\S}$ (Figure) and was at or slightly above the region-specific baseline in four of the 10 U.S. Department of Health and Human Services regions (Regions 4 and 7-9). The majority of jurisdictions experienced minimal or low ILI activity since September 30; however, two experienced moderate ILI activity, and two experienced high ILI activity** during the week ending December 1 . The percentage of deaths attributed to pneumonia and influenza remains below the epidemic threshold, ${ }^{\dagger \dagger}$ and the rate of influenza-associated hospitalizations remains low. Five laboratory-confirmed,

\footnotetext{
* Data as of December 7, 2018.

$\dagger$ Defined as a fever (temperature $\geq 100^{\circ} \mathrm{F}\left[\geq 37.8^{\circ} \mathrm{C}\right]$, oral or equivalent) and cough or sore throat, without a known cause other than influenza.

$\$$ The national and regional baselines are the mean percentages of visits for ILI during noninfluenza weeks for the previous three seasons plus two standard deviations. Noninfluenza weeks are defined as periods of $\geq 2$ consecutive weeks in which each week accounted for $<2 \%$ of the season's total number of specimens that tested positive for influenza in public health laboratories. National and regional percentages of patient visits for ILI are weighted based on state population. Use of the national baseline for regional data is not appropriate.

I Region 1: Connecticut, Maine, Massachusetts, New Hampshire, Rhode Island, and Vermont; Region 2: New Jersey, New York, Puerto Rico, and U.S. Virgin Islands; Region 3: Delaware, District of Columbia, Maryland, Pennsylvania, Virginia, and West Virginia; Region 4: Alabama, Florida, Georgia, Kentucky, Mississippi, North Carolina, South Carolina, and Tennessee; Region 5: Illinois, Indiana, Michigan, Minnesota, Ohio, and Wisconsin; Region 6: Arkansas, Louisiana, New Mexico, Oklahoma, and Texas; Region 7: Iowa, Kansas, Missouri, and Nebraska; Region 8: Colorado, Montana, North Dakota, South Dakota, Utah, and Wyoming; Region 9: Arizona, California, Hawaii, Nevada, American Samoa, Commonwealth of the Northern Mariana Islands, Federated States of Micronesia, Guam, Marshall Islands, and Republic of Palau; Region 10: Alaska, Idaho, Oregon, and Washington.

** Activity levels are based on the percentage of outpatient visits in a jurisdiction attributed to ILI and are compared with the average percentage of ILI visits that occur during weeks with little or no influenza virus circulation. Activity levels range from minimal, corresponding to ILI activity from outpatient clinics at or below the average, to high, corresponding to ILI activity from outpatient clinics much higher than the average. Because the clinical definition of ILI is nonspecific, not all ILI is caused by influenza; however, when combined with laboratory data, the information on ILI activity provides a clearer picture of influenza activity in the United States.

$\dagger \dagger$ The seasonal baseline proportion of pneumonia and influenza deaths is projected using a robust regression procedure, in which a periodic regression model is applied to the observed percentage of deaths from pneumonia and influenza that were reported by the National Center for Health Statistics Mortality Surveillance System during the preceding 5 years. The epidemic threshold is set at 1.645 standard deviations above the seasonal baseline.
}

influenza-associated pediatric deaths occurring since September 30 have been reported to CDC. During the week ending December 1, the majority of jurisdictions (40 states, the District of Columbia, Puerto Rico, and U.S. Virgin Islands) reported sporadic or local geographic spread of influenza activity, nine states reported regional activity, and one state reported widespread activity. $\$ \$$

Influenza $\mathrm{A}(\mathrm{H} 1 \mathrm{~N} 1) \mathrm{pdm} 09$ viruses have been reported most frequently $(67 \%$ of all viruses and $81 \%$ of subtyped influenza A viruses) by U.S. public health laboratories since September 30, 2018 (Table), but A(H3N2) and influenza B viruses also were reported. The majority of influenza viruses characterized during this period were genetically and antigenically similar to the cell-grown reference viruses representing the 2018-19 Northern Hemisphere influenza vaccine viruses. 99 No viruses with resistance to oseltamivir, zanamivir, or peramivir have been identified.

The timing of influenza activity often varies; however, influenza activity will increase in coming weeks and is likely to peak during December-February. Annual influenza vaccination is recommended for all persons aged $\geq 6$ months who do not have contraindications (1). Multiple influenza vaccines are approved and recommended for use during the 2018-19 season, and vaccination should continue to be offered as long as influenza viruses are circulating and unexpired vaccine is available. For the 2018-19 season, manufacturers projected they would supply the United States with 163-168 million doses

\footnotetext{
$\$ \$$ Levels of activity are 1) no activity; 2) sporadic: isolated laboratory-confirmed influenza cases or a laboratory-confirmed outbreak in one institution, with no increase in activity; 3) local: increased ILI or two or more institutional outbreaks (ILI or laboratory-confirmed influenza) in one region of the state, with recent laboratory evidence of influenza in that region; virus activity no greater than sporadic in other regions; 4) regional: increased ILI activity or institutional outbreaks (ILI or laboratory-confirmed influenza) in two or more outbreaks, but less than half of the regions in the state with recent laboratory evidence of influenza in those regions; and 5) widespread: increased ILI activity or institutional outbreaks (ILI or laboratory-confirmed influenza) in at least half the regions in the state, with recent laboratory evidence of influenza in the state. During the week ending December 1, 2018, nine states (California, Connecticut, Georgia, Kentucky, Louisiana, Nevada, New York, Oregon, and Vermont) reported regional activity, and one state (Massachusetts) reported widespread activity.

99 The recommended Northern Hemisphere 2018-19 trivalent influenza vaccine composition includes an A/Michigan/45/2015 (H1N1)pdm09-like virus, an A/Singapore/INFIMH-16-0019/2016 (H3N2)-like virus, and a B/Colorado/06/2017-like (Victoria lineage virus), with an additional influenza B virus (B/Phuket/3073/2013-like [Yamagata lineage]) virus recommended for quadrivalent vaccines.
} 
FIGURE. Percentage of visits for influenza-like illness (ILI) - U.S. Outpatient Influenza-like Illness Surveillance Network (ILINet), weekly national summary, 2018-2019* and selected previous seasons

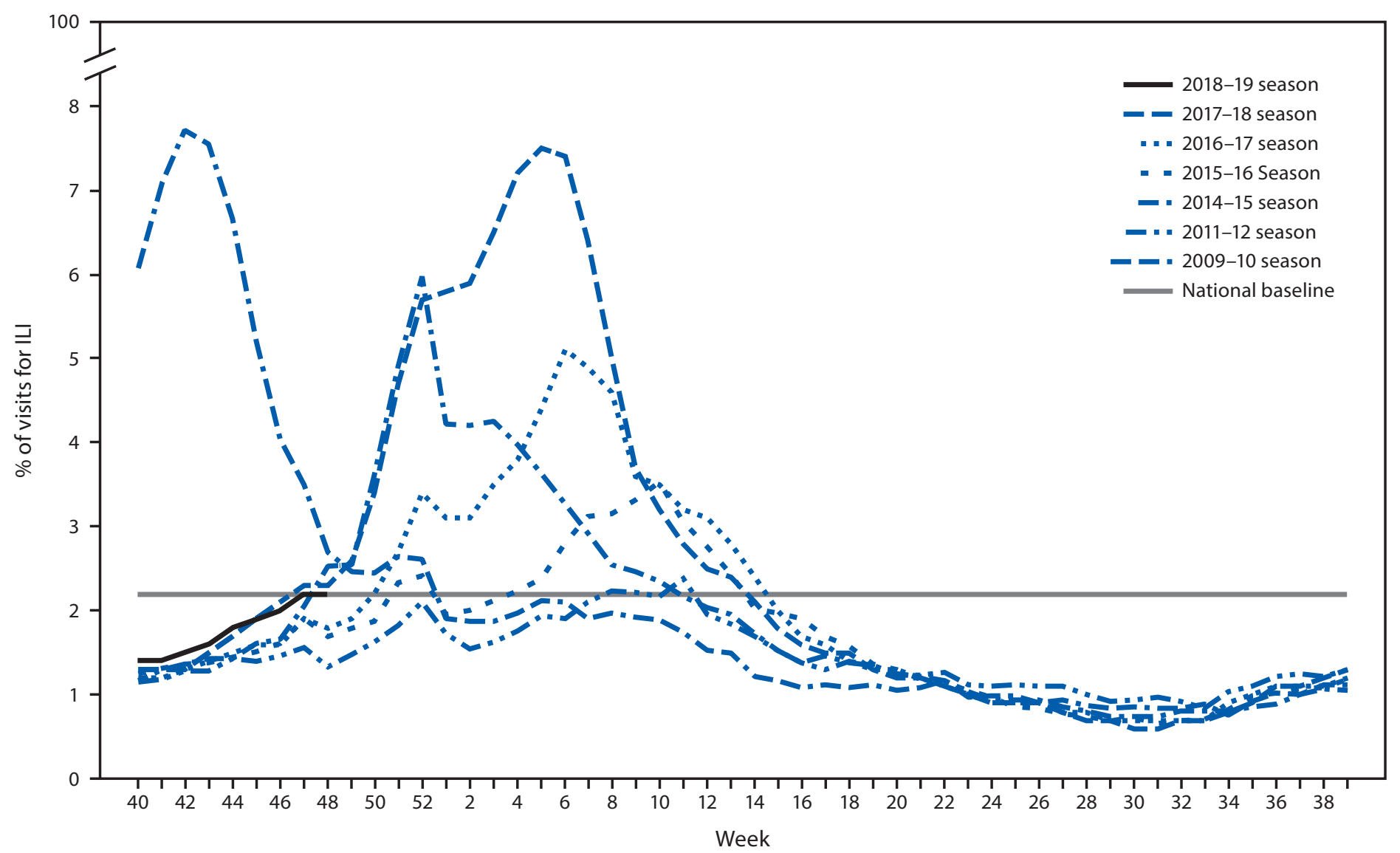

* As of December 7, 2018.

TABLE. Influenza positive specimens reported by U.S. public health laboratories - United States, September 30-December 1, 2018*

\begin{tabular}{lc}
\hline Influenza virus type/Subtype or lineage & $\begin{array}{c}\text { No. of positive specimens } \\
\text { (\% of total) }\end{array}$ \\
\hline Influenza A viruses & $740(67)$ \\
Influenza A(H1N1)pdm09 & $176(16)$ \\
Influenza A(H3N2) & $92(8)$ \\
Influenza A (subtyping not performed) & \\
Influenza B viruses & $60(5)$ \\
Influenza B Yamagata & $21(2)$ \\
Influenza B Victoria & $22(2)$ \\
Influenza B (lineage not performed) &
\end{tabular}

* As of December 7, 2018.

of influenza vaccine. As of November 30, 2018, approximately 163.8 million doses had been distributed.

Influenza antiviral medications are an important adjunct to vaccination in the treatment and prevention of influenza. There are four recommended influenza antiviral medications for treatment of influenza this season: oral oseltamivir, inhaled zanamivir, intravenous peramivir, and the newly approved oral baloxavir. Treatment with influenza antiviral medications as close to the onset of illness as possible is recommended for patients with confirmed or suspected influenza who have severe, complicated, or progressive illness; who require hospitalization; or who are at high risk for influenza complications. Some antiviral medications (oseltamivir and zanamivir) can be considered for chemoprophylaxis to prevent influenza in certain situations; however, general seasonal or preexposure antiviral chemoprophylaxis is not recommended. Updated recommendations for use of antiviral drugs are available (https://www.cdc.gov/flu/professionals/antivirals/summaryclinicians.htm).

Influenza surveillance reports for the United States are posted online weekly (https://www.cdc.gov/flu/weekly). Additional information regarding influenza viruses, influenza surveillance, influenza vaccines, and influenza antiviral medications is available online (https://www.cdc.gov/flu).

\section{Acknowledgments}

State, county, city, and territorial health departments and public health laboratories; U.S. World Health Organization collaborating laboratories; National Respiratory and Enteric Virus Surveillance System laboratories; U.S. Outpatient Influenza-Like Illness 
Surveillance Network sites; FluSurv-NET; the National Center for Health Statistics, CDC; LaShondra Berman, Elisabeth Blanchard, Priya Budhathoki, Roxana Cintron, Juliana DaSilva, Juan De la Cruz, Angie Foust, Lizheng Guo, Norman Hassell, Shoshona Le, Ji Liu, Brian Lynch, Vasiliy Mishin, Janná Murray, Ha Nguyen, Thomas Rowe, Sujatha Seenu, Samuel Shepard, Bo Shu, Catherine Smith, Thomas Stark, Alma Trujillo, Malania Wilson, Influenza Division, National Center for Immunization and Respiratory Diseases, CDC.

Corresponding author: Alicia Budd, abudd@cdc.gov, 404-639-3747.

\footnotetext{
${ }^{1}$ Influenza Division, National Center for Immunization and Respiratory Diseases, CDC.
}

The authors have completed and submitted the ICMJE form for disclosure of potential conflicts of interest. No potential conflicts of interest were disclosed.

\section{Reference}

1. Grohskopf LA, Sokolow LZ, Broder KR, Walter EB, Fry AM, Jernigan DB. Prevention and control of seasonal influenza with vaccines: recommendations of the Advisory Committee on Immunization Practices-United States, 2018-19 influenza season. MMWR Recomm Rep 2018;67(No. RR-3). https://doi.org/10.15585/mmwr.rr6703a1 